\title{
10 años de Proyecto Ríos en Cantabria: resultados, logros, lecciones y oportunidades
}

\author{
GRACIA, A. ${ }^{*} ;$ VALDOR, P.F. ; CLOUX, I. ${ }^{1}$ TEJÓN, S. ${ }^{1}$ \\ ${ }^{1}$ Red Cambera, Apdo. Correos 4013, CP 39011. Peñacastillo, Santander, Cantabria. * redcambera@gmail.com
}

\section{INFORMACIÓN DEL ARTÍCULO ${ }^{1}$}

Recibido el 3 de noviembre de 2019

Revisión recibida el 29 de mayo de 2020

Aceptado el 4 de junio de 2020

DOI: $10.51443 /$ Restaurarios.2020.02

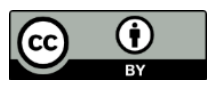

\author{
Palabras clave \\ Conservación \\ Capacitación \\ Red Cambera \\ Voluntariado ambiental.
}

\begin{abstract}
Resumen
El Proyecto Ríos es un programa de participación ciudadana para el diagnóstico y mejora de los ríos basado en ciencia ciudadana, educación ambiental, voluntariado ambiental y custodia del territorio. La metodología del proyecto comprende 5 fases: compromiso de las personas voluntarias, capacitación en el uso de metodologías científicas para la recopilación de datos ambientales y la aplicación de índices, inspección de un tramo de río de unos 500 metros de longitud dos veces al año (primavera y otoño), diagnóstico de la salud del tramo fluvial y adopción del tramo de río para el desarrollo de acciones de conservación. Tras la implementación durante 10 años del Proyecto Ríos en Cantabria se ha realizado un análisis estadístico descriptivo de la información ambiental recabada por las personas voluntarias y una recopilación de las acciones sociales y ambientales desarrolladas. Los resultados muestran un total de 282 tramos de río inspeccionados y alrededor de 300 acciones desarrolladas. Durante el periodo de estudio, el Proyecto Ríos ha demostrado ser una herramienta fundamental para involucrar a la ciudadanía en el estudio y cuidado de los ríos de Cantabria. Asimismo, ha permitido generar datos útiles en la toma de decisiones por parte de las asociaciones conservacionistas, la comunidad científica y la Administración.
\end{abstract}

Keywords

Conservation

Training

Red Cambera

Environmental volunteering

\begin{abstract}
Proyecto Ríos is a citizen participation program, whose purpose is to survey Cantabrian rivers, based on citizen's science, environmental education, environmental volunteering and land custody. The methodology is based on 5 stages: to take on the commitment of surveying a river section, to train volunteers on scientific methodologies to survey a river section, to inspect of a 500 meters river section twice per year (on spring and autumn) by volunteering people, to diagnose the ecological status of the river systems and to adopt a river stretch by volunteering people. After 10 years, Red Cambera has carried out a statistical analysis of environmental data and a review of social and environmental activities developed. In this study, the main results obtained are presented. The main results of this study shown that 282 river sections were surveyed by volunteering people and around 300 social and environmental activities were developed. During the study period, Proyecto Ríos has demonstrated to be a fundamental tool for involving citizenship on the study and care of river systems located in Cantabria. Furthermore, Proyecto Ríos is recognized as a tool to generate useful data which could be an extra support for make decisions by conservation associations, the scientific community and public administrations.
\end{abstract}

\section{Introducción y objetivos}

El concepto de educación ambiental fue nombrado por primera vez en la reunión fundacional de la UICN en los años 50 (Alonso, 2010). Si bien, el término no fue definido hasta el año 1970 en la Carta de Nevada (UICN, 1970). A partir de la Conferencia Intergubernamental de Tbilisi sobre educación ambiental en 1977, dicha educación se comenzó a considerar como una herramienta para fomentar el proceso activo para la mejora de la vida y la protección del medio ambiente. Por otro lado, la ciencia 
ciudadana se define como aquella que involucra a todos los públicos en actividades científicas y fomenta la participación activa de este sector en la investigación (Serrano et al., 2014). De esta manera, la ciencia ciudadana es una herramienta abierta, participativa, voluntaria y transversal, que permite que los ciudadanos y ciudadanas contribuyan a la ciencia activamente: aportando datos, produciendo nuevos resultados o permitiendo la difusión del conocimiento. Además, por medio de la ciencia ciudadana se establecen vínculos entre investigadores e investigadoras, ciudadanos y ciudadanas y el medio ambiente, lo que permite la participación pública en la producción de conocimiento científico. Por tanto, la educación ambiental y la ciencia ciudadana están estrechamente relacionadas procurando, en ambos casos, un aprendizaje basado en la inquietud y las ganas de aprender de las personas. En este contexto de aprendizaje participativo se enmarca el Proyecto Ríos.

El Proyecto Ríos es un programa de ciencia ciudadana, educación ambiental, voluntariado y custodia del territorio que se basa en las ganas de hacer y el altruismo de la ciudadanía. Su fin es lograr que los ciudadanos y ciudadanas, en equipo, participen en la generación de conocimiento sobre el estado de los ríos y riberas, así como que se involucren en la conservación y mejora del medio ambiente. El proyecto comenzó como una iniciativa de la Associació Hàbitats (Cataluña) en el año 1998. Actualmente, existen diversas entidades que lo desarrollan en otros territorios de la Península Ibérica: la Asociación para a Defensa Ecolóxica de Galiza (Galicia), la Fundación Limne (Demarcación Hidrográfica del Júcar), la Asociación Territorios Vivos (Madrid) y la Asociación Portuguesa de Educación Ambiental (Portugal). Todas ellas forman la Red Ibérica de Proyecto Ríos. En Cantabria, el Proyecto Ríos se inició en el año 2008, en el Centro de Investigación del Medio Ambiente (CIMA), organismo autónomo del Gobierno de Cantabria. Sin embargo, desde el año 2010 se coordina a través de la asociación Red Cambera. Red Cambera es una entidad sin ánimo de lucro e independiente que trabaja con el objetivo principal de conservar el medio natural. Sus líneas de acción son cuatro: ciencia ciudadana, custodia del territorio, educación ambiental y participación activa.

El objetivo de este trabajo es mostrar la utilidad práctica de un proyecto de voluntariado, tanto para la ciencia como para la sociedad en general, para el seguimiento ambiental de los ríos y riberas, así como para el fomento de la sensibilización y la educación ambiental. Como objetivos específicos se considera: la evaluación de la calidad biológica del agua, del estado del bosque de ribera y del estado ecológico de los tramos de ríos muestreados en el marco del Proyecto Ríos 2008- 2017; la validación de las valoraciones de la calidad biológica del agua obtenidas por personal experto en la materia y las realizadas por las personas voluntarias del Proyecto Ríos; la identificación de tramos inspeccionados con un gran valor ecológico o que presenten retos ambientales; y la exposición de las acciones sociales emprendidas.

\section{Metodología}

A continuación, se describen brevemente las metodologías empleadas en el Proyecto Ríos, un análisis descriptivo de los datos ambientales recabados por las personas voluntarias y una recopilación de las acciones desarrolladas en el marco del Proyecto Ríos Cantabria (2008-2017).

\section{$2.1 \quad$ Proyecto Ríos}

El Proyecto Ríos está dirigido a cualquier colectivo o persona que quiera participar de manera voluntaria, ya sean centros educativos, asociaciones, familias, grupos de amigos o amigas, o ciudadanos y ciudadanas de manera individual. La metodología se estructura en 5 fases:

La primera fase consiste en el establecimiento de un compromiso entre el grupo de personas voluntarias y Red Cambera. Las personas voluntarias eligen, de manera libre, un tramo de río de cualquier cuenca cántabra que cumpla con las siguientes características: presentar una longitud de unos 500 metros, ser de agua dulce y no estar comprendido dentro de zonas estuarinas y/o masas de aguas lénticas.

La segunda etapa comprende la capacitación del voluntariado. En las fechas previas a cada campaña de inspección, se realizan jornadas formativas de 2- 3 horas de duración en zonas accesibles de un río cántabro. Estas jornadas están destinadas a los nuevos grupos de voluntarios y voluntarias. En ellas, se les entrega un kit de inspección de ríos. Dicho kit contiene: ficha de campo, manual de inspección de ríos, claves de identificación de flora y fauna, fichas para el cálculo de índices de calidad ambiental, red para la recogida de macroinvertebrados bentónicos, termómetro, disco de Secchi (o de transparencia) y lupa. Durante la jornada se les explica los conceptos ambientales básicos, el manejo de los materiales entregados (uso de la red para la recogida de macroinvertebrados y disco de Secchi), el cálculo de índices de calidad ambiental (Índice del Hábitat Fluvial (IHF), estado del bosque de ribera (QRISI) y calidad biológica del agua mediante macroinvertebrados (IBMWP) y cómo realizar el inventario de especies autóctonas y alóctonas.

La tercera etapa consiste en la caracterización de un tramo de río mediante la toma de datos in situ. Para ello, las personas voluntarias acuden al río dos veces al año 
(primavera y otoño) para completar la ficha de campo, donde registran información relativa a: la inspección básica del tramo (condiciones de las márgenes, usos de los suelos y presencia de residuos), la inspección de vertidos, el hábitat fluvial (grado de inclusión de cantos y gravas en rápidos y pozas, frecuencia de rápidos, composición del sustrato, regímenes de velocidad y profundidad, elementos de heterogeneidad y cobertura de vegetación acuática) y el estudio del ecosistema acuático (temperatura, transparencia, anchura, profundidad, velocidad, caudal, presencia de especies de flora y fauna tanto autóctona como alóctona, estudio de macroinvertebrados y características del bosque de ribera).

La cuarta etapa comprende el diagnóstico de la salud del tramo fluvial. Con la información obtenida en la tercera fase, las personas voluntarias realizan un diagnóstico completo y estandarizado del tramo seleccionado. El diagnóstico está compuesto por tres valoraciones: calidad biológica del agua, estado del bosque de ribera y la integración de las mismas en el estado ecológico.

Tal y como se ha mencionado, la calidad biológica del agua se estima según el indicador basado en macroinvertebrados acuáticos adaptado del índice IBMWP (Iberian Bio-Monitoring Working Party) (AlbaTercedor y Sánchez Ortega, 1988). La diversidad y abundancia de organismos en un río puede ser indicativo del estado de salud en el que se encuentra. El índice de calidad biológica del agua se aplica en 100 metros del tramo total de río escogido, donde se recogen 10 submuestras en los distintos hábitats más representativos de la zona. A partir de los macroinvertebrados bentónicos recolectados e identificados a nivel de familia se calcula la calidad biológica del agua. La calidad se estima mediante una ficha-guía que atiende a las familias de macroinvertebrados identificadas en la muestra recogida y su abundancia (Cloux et al., 2018) y recoge los diferentes estados de calidad del agua de forma jerarquizada a través de un código de colores.

Por su parte, la calidad del bosque de ribera se determina mediante el índice QRISI que es una simplificación del Índice de Calidad del Bosque de Ribera (QBR) (Suárez et al., 2002). El índice QRISI se aplica en 100 metros del tramo total de río escogido. El índice sirve para caracterizar de manera rápida el estado de conservación de nuestras riberas con independencia de las especies vegetales presentes. Mediante una ficha-guía (Cloux et al., 2018), se valoran de manera visual 3 parámetros que hacen referencia al bosque de ribera: estructura y complejidad de la ribera (en función del mayor o menor grado de cobertura vegetal en base a los estratos de vegetación presentes), conexión con formas vegetales adyacentes y continuidad de la vegetación de ribera a lo largo del río.

Finalmente, para la determinación del estado ecológico, se utiliza una adaptación del índice ECOSTRIMED (ECOlogical STatus RIver MEDiterranean) (Prat et al., 2000) que integra el índice biológico de calidad del agua y el índice de calidad del bosque de ribera (Tabla 1). Todos estos índices simplificados han sido recientemente aplicados a los ecosistemas fluviales de Cantabria por Cloux et al. (2018) y Cloux et al. (2019).

Tabla 1 Cálculo del estado ecológico del río a partir de los resultados del Índice biológico de calidad del agua y del Índice de calidad del bosque de ribera (QRISI).

\begin{tabular}{|c|c|c|c|}
\hline \multirow[b]{2}{*}{$\begin{array}{l}\text { Índice } \\
\text { biológico de } \\
\text { calidad del } \\
\text { agua }\end{array}$} & \multicolumn{3}{|c|}{$\begin{array}{l}\text { Índice de calidad del bosque de ribera } \\
\text { (QRISI) }\end{array}$} \\
\hline & $\begin{array}{c}\text { Estado } \\
\text { óptimo. } \\
\text { Bien } \\
\text { conservado }\end{array}$ & $\begin{array}{l}\text { Alteraciones } \\
\text { importantes }\end{array}$ & $\begin{array}{c}\text { Muy } \\
\text { degradado }\end{array}$ \\
\hline Миу bиепо & $\begin{array}{l}\text { Muy buen } \\
\text { estado }\end{array}$ & Buen estado & Moderado \\
\hline Bиепо & Buen estado & Moderado & Deficiente \\
\hline Moderado & Moderado & Deficiente & Malo \\
\hline Deficiente & Deficiente & Malo & Malo \\
\hline Malo & Malo & Malo & Malo \\
\hline
\end{tabular}

La quinta y última fase consiste en la adopción del tramo de río y se lleva a cabo cuando las personas voluntarias tienen la inquietud de desarrollar actuaciones complementarias para resolver las necesidades ambientales que presenta su tramo. La adopción es un modelo de custodia del territorio basado en el acuerdo de todas las partes implicadas en la gestión de un tramo concreto de río, teniendo en cuenta que se encuentran dentro del Dominio Público Hidráulico y/ o la zona de policía (Tabla 2), con el fin de su conservación o mejora. Esta última fase es, por tanto, una vía de participación activa en la conservación del patrimonio común y una forma de implicación social voluntaria en la que los ciudadanos y ciudadanas son los protagonistas, promotores y ejecutores de las actuaciones de conservación del ecosistema fluvial.

\subsection{Análisis descriptivo de los datos ambientales}

Para llevar a cabo el análisis de los datos recabados en el marco del Proyecto Ríos durante el periodo 2008-2017, se definen dos escalas espaciales de trabajo: una escala regional (Cantabria) y una escala a nivel de punto de muestreo. En lo que se refiere al análisis a escala regional, las valoraciones se realizan para cada uno de los puntos de muestreo en periodos mínimos anuales, así como para todo el periodo comprendido entre 2008 y 2017. Para dicho periodo, la calidad biológica del agua, el estado del bosque de ribera y el estado ecológico son valorados mediante el cálculo del valor promedio de las valoraciones anuales. En función del punto de muestreo 
Tabla 2 Ámbito competencial de las actuaciones de mejora y conservación de cauces. (Fuente: Ministerio para la Transición Ecológica, 2019).

\section{ORGANISMOS DE CUENCA}

Ámbito competencial

Las actuaciones en cauce fuera de suelo urbano o actuaciones en cauces no ordinarias en tramo urbano sujetas a convenio o por iniciativa del Organismo de cuenca.

\section{Normativa}

Texto Refundido de la Ley de Aguas.

Reglamento del Dominio Público

Hidráulico (art. 126 y 126bis).

Real Decreto 903/2010, de 9 de julio, de evaluación y gestión de riesgos de inundación (apart. I.h) Anexo I).

Real Decreto 984/1989, de 28 de julio, por

el que se determina la estructura orgánica

dependiente de la Presidencia de las

Confederaciones Hidrográficas (art. 4.k).

\section{Actuaciones}

Cualquier actuación en cauce en función de las prioridades, salvo la retirada de residuos sólidos urbanos y tratamientos de vegetación en cauce para la prevención de incendios.

\section{ENTIDADES LOCALES Y CC. AA}

\section{Ámbito competencial}

Las actuaciones en espacios materialmente urbanos. La retirada de residuos sólidos urbanos en cauce y el tratamiento de vegetación en cauces y zona de policía para la prevención de incendios. Si estas afectaran a la vegetación en cauce o zona de policía o a la morfología del cauce, requieren autorización del Organismo de cuenca.

\section{Normativa}

Ley de Bases de Régimen Local (art. 25, 26 y 36$)$.

Ley 10/2001 del Plan Hidrológico

Nacional (art. 28.4).

Ley 22/2011 de residuos y suelos

contaminados (art. 12.5.a)

STS 4626/2017 y STS 1489/2012.

\section{Actuaciones}

Actuaciones ordinarias en cauces: Retirada de residuos asimilables a urbanos (basuras, escombros, restos de mobiliario, etc.). Mantenimiento de la vegetación de ribera, que por su densidad pueda dar lugar a tapones, malos olores encharcamientos, etc. Obras de protección frente a riesgos de inundación en espacios urbanos. Tratamientos de vegetación en cauce para evitar la propagación de incendios, cuando proceda.

\section{OTROS SOLICITANTES (persona física, jurídica o administración)}

Ámbito competencial

En todo caso requerirán autorización del

Organismo de cuenca

\section{Normativa}

Texto Refundido de la Ley de Aguas.

Real Decreto 984/1989, de 28 de julio, por el que se determina la estructura orgánica dependiente de la Presidencia de las Confederaciones Hidrográficas.

Reglamento del Dominio Público

\section{Actuaciones}

Para cualquier tipo de actuación en cauce, ya sea dentro o fuera de espacio urbano.

Hidráulico.

del que se trate, el periodo analizado varía entre 1 y 10 años. Una vez estimados, se representan espacialmente en forma de mapa.

A modo de ejercicio de validación, se lleva a cabo un análisis comparativo entre las valoraciones de la calidad biológica del agua obtenidas por personal experto en la materia y las realizadas por las personas voluntarias del Proyecto Ríos. Para ello, se seleccionan los puntos de muestreo de la Red MARCE (IH Cantabria, 2012) localizados a una distancia menor o igual a 100 metros respecto a los puntos de muestreo inspeccionados en el marco del Proyecto Ríos (2008-2017). Una vez seleccionados, se estima la calidad biológica del agua para cada uno de los puntos de muestreo. Por último, con el objetivo de conocer el grado de semejanza entre las valoraciones obtenidas tras la aplicación de ambos procedimientos (Red MARCE vs Proyecto Ríos), se calcula el valor del índice Kappa ponderado (Cohen, 1960; Cohen, 1968). Este valor es un número real adimensional comprendido entre $-1 \mathrm{y}+1$. El grado de acuerdo entre las valoraciones de ambos procedimientos será mayor cuanto mayor sea el valor del índice Kappa (Tabla 3).

Finalmente, se categorizan los tramos de gran valor ecológico y los tramos que presentan retos ambientales. Estos últimos, son los tramos sobre los que aplicar futuras líneas de acción para lograr una conservación y mejora del estado de los ríos y riberas de Cantabria. Los tramos identificados como retos ambientales son aquellos que presentan un estado ecológico inferior a bueno, o un 
estado ecológico bueno como resultado de una calidad biológica del agua muy buena y un bosque con alteraciones importantes. Los tramos identificados como de gran valor ecológico son aquellos que presentan un estado ecológico muy bueno o bueno (Tabla 4).

En lo que se refiere el análisis a escala de punto de muestreo, se identifican los puntos muestreados durante un periodo de al menos 6 años con el objetivo de mostrar la evolución temporal del estado ecológico de cada uno de ellos para el periodo 2008 -2017.

Tabla 3 Correspondencias entre el valor del índice Kappa ponderado (K) y el grado de acuerdo (Fuente: Bald, 2005).

\begin{tabular}{ll}
\hline Valor de K & Grado de acuerdo \\
$0-0.05$ & Nulo \\
$0.05-0.20$ & Muy bajo \\
$0.20-0.40$ & Bajo \\
$0.40-0.55$ & Moderado \\
$0.55-0.70$ & Bueno \\
$0.70-0.85$ & Muy Bueno \\
$0.85-0.99$ & Prácticamente perfecto \\
$0.99-1.00$ & Perfecto \\
\hline
\end{tabular}

Tabla 4 Clasificación del estado ecológico, significado y categorización de los tramos.

\begin{tabular}{|c|c|c|}
\hline $\begin{array}{l}\text { Estado } \\
\text { ecológico }\end{array}$ & Significado & Tramos \\
\hline $\begin{array}{l}\text { Muy buen } \\
\text { estado }\end{array}$ & $\begin{array}{l}\text { Ríos sin alteración } \\
\text { humana }\end{array}$ & $\begin{array}{l}\text { Alto valor ecológico } \\
\text { Oportunidades: }\end{array}$ \\
\hline Buen estado & Leves alteraciones & $\begin{array}{l}\text { Conservación } \\
\text { ambiental }\end{array}$ \\
\hline $\begin{array}{l}\text { Estado } \\
\text { moderado }\end{array}$ & $\begin{array}{l}\text { Alteraciones } \\
\text { considerables }\end{array}$ & Reto ambiental \\
\hline $\begin{array}{l}\text { Estado } \\
\text { deficiente }\end{array}$ & $\begin{array}{l}\text { Alteraciones } \\
\text { importantes }\end{array}$ & $\begin{array}{l}\text { Retos: Restauración } \\
\text { ambiental }\end{array}$ \\
\hline Estado malo & Muy degradado & \\
\hline
\end{tabular}

2.3 Recopilación de las acciones desarrolladas en el marco del Proyecto Ríos Cantabria (2008-2017)

A continuación, se definen los distintos tipos de acciones sociales y ambientales emprendidas:

\subsubsection{Acciones formativas}

Entre las que se incluyen: $i$ ) salidas formativas: en las fechas previas a cada campaña de inspección los nuevos grupos, acompañados por personal técnico de Red Cambera, realizan salidas de campo para la inspección y diagnóstico de un tramo de río; ii) muestreos simultáneos: donde se diagnostica el estado de varios tramos de río en un mismo día por varios grupos de personas voluntarias; iii) conferencias/jornadas formativas: llevadas a cabo por especialistas en diferentes temáticas relacionadas con el medio fluvial (flora exótica, aguas subterráneas, patrimonio fluvial, etc.). Las jornadas están orientadas a la formación en la identificación de macroinvertebrados fluviales en laboratorio y el reconocimiento de huellas y excrementos de mamíferos en el entorno fluvial; iv) itinerarios interpretativos: orientados a la interpretación de los sistemas fluviales.

\subsubsection{Actividades de encuentro entre personas voluntarias}

Entre las que se incluyen, las redes de voluntarios que son una herramienta participativa con la que reunir a grupos de voluntarios de una misma cuenca en un determinado lugar de la misma para evaluar el presente y realizar nuevas propuestas de actuación para el futuro. Y las romerías fluviales que son reuniones lúdico-formativas donde se reúnen todos los voluntarios y voluntarias del Proyecto Ríos con la intención de agradecerles el esfuerzo realizado y fomentar su conocimiento y relación.

\subsubsection{Acciones de custodia fluvial y adopción de ríos}

Las acciones de custodia fluvial se encuentran enmarcadas dentro de la denominada custodia del territorio, que es un conjunto de estrategias e instrumentos que pretenden implicar a los propietarios de los predios colindantes y usuarios del territorio en la conservación y el buen uso de los valores y los recursos naturales, culturales y paisajísticos. Mientras que las acciones de adopción de ríos son aquellas encaminadas a desarrollar propuestas de los grupos de voluntarios que impliquen la mejora o el aumento del conocimiento de los ambientes fluviales, esto es, realizar actuaciones prácticas que ayuden al río a alcanzar su estado óptimo. En ambos casos, supone un compromiso con el entorno natural a largo plazo.

\subsubsection{Acciones de conservación}

Son aquellas organizadas de manera puntual por la entidad, sin necesidad de la firma de un acuerdo, en una determinada zona destinadas a la concienciación y sensibilización ambiental de los ciudadanos y ciudadanas, y cuyo contenido puede abarcar diversas temáticas.

\section{Resultados}

\subsection{Resultados del análisis de los datos ambientales}

Durante el periodo 2008-2017 se realizaron un total de 1.482 inspecciones y se diagnosticaron un total de 282 tramos de río repartidos por 12 cuencas fluviales cántabras, de los que 157 se encontraron dentro de Zonas de Especial Conservación Fluviales de Cantabria. Respecto a los resultados del análisis a escala regional, alrededor del $70 \%$ de los tramos presentaron una calidad biológica del agua buena o muy buena. Estos puntos se localizaron principalmente en los tramos medios y altos de los principales ríos de la región. En la Figura 1, se muestra la distribución espacial de los valores promedio 


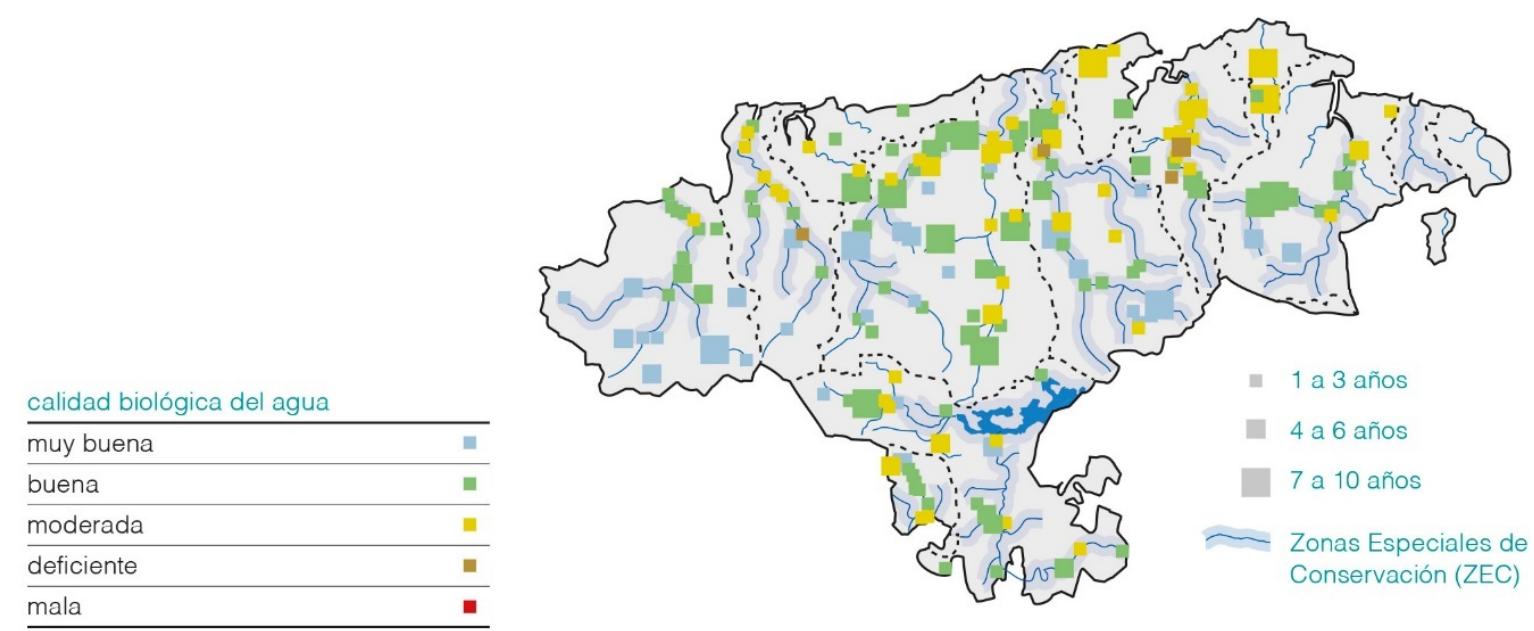

Figura 1 Calidad biológica del agua en los puntos de muestreo diagnosticados durante el periodo 2008-2017 en el marco del Proyecto Ríos Cantabria.

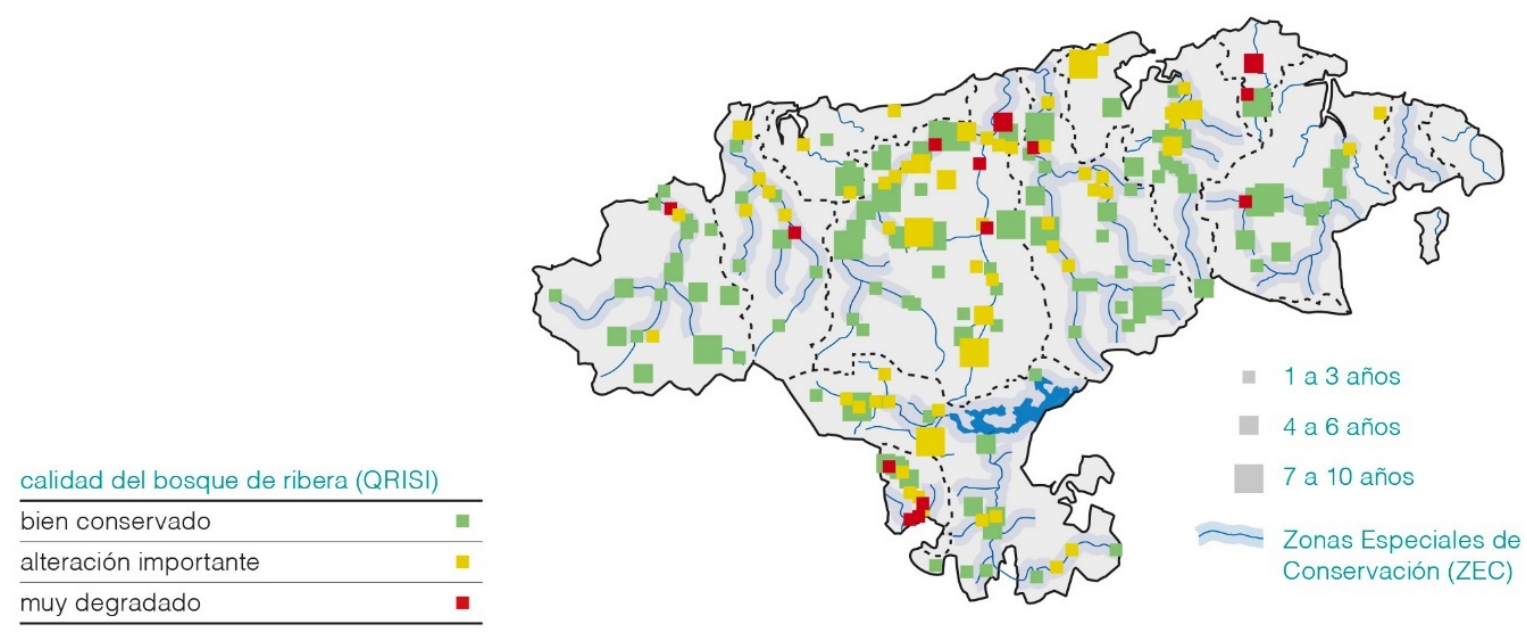

Figura 2 Calidad del bosque de ribera en los puntos de muestreo diagnosticados durante el periodo 2008 -2017 en el marco del Proyecto Ríos Cantabria.

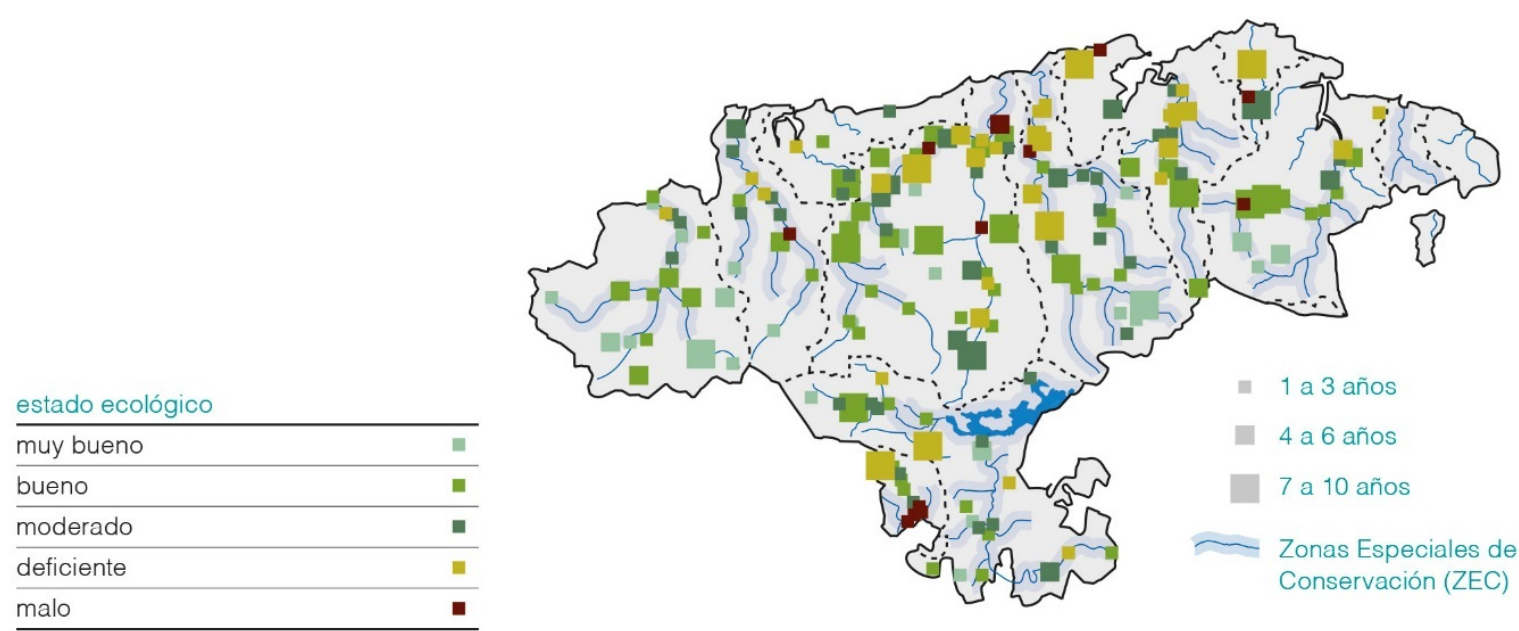

Figura 3 Estado ecológico promedio en los puntos diagnosticados en el marco del Proyecto Ríos. 
de la calidad biológica del agua. En función del punto de muestreo del que se trata, el periodo analizado para cada tramo varía entre 1 y 10 años. En torno al $63 \%$ de los tramos inspeccionados presentaron un bosque de ribera en estado óptimo y bien conservado. Estos tramos se localizaron principalmente en los tramos altos de los ríos, y en los tramos medios y bajos de las cuencas con menor presión antrópica (Figura 2). Alrededor de la mitad de los tramos presentaron un estado ecológico bueno o muy bueno (49.5\%). Los puntos con un estado ecológico bueno o muy bueno se localizaron principalmente en zonas de cabecera y medias de los ríos, mientras que los puntos con un estado moderado o inferior se asociaron con zonas más próximas a los tramos medios y bajos de los ríos (Figura 3).
En la Figura 4 se muestran los puntos de muestreo de la Red MARCE localizados a una distancia menor o igual a 100 metros respecto a los puntos de muestreo de la Red Proyecto Ríos. Los 7 puntos coincidentes fueron muestreados en 43 ocasiones por ambas redes (Red MARCE y Proyecto Ríos), no siendo coincidentes en el tiempo las campañas de muestreo realizadas por cada una de las redes. Los valores de calidad biológica del agua obtenidos en el diagnóstico de las 43 campañas realizadas por ambas redes en los puntos de muestreo seleccionados fueron comparados para obtener el valor del índice Kappa ponderado. El valor del índice Kappa resultado del análisis comparativo fue de 0.63 , lo cual indicaba un grado de acuerdo Bueno entre las valoraciones de la calidad biológica del agua obtenidas por ambas redes (Tabla 3 ).
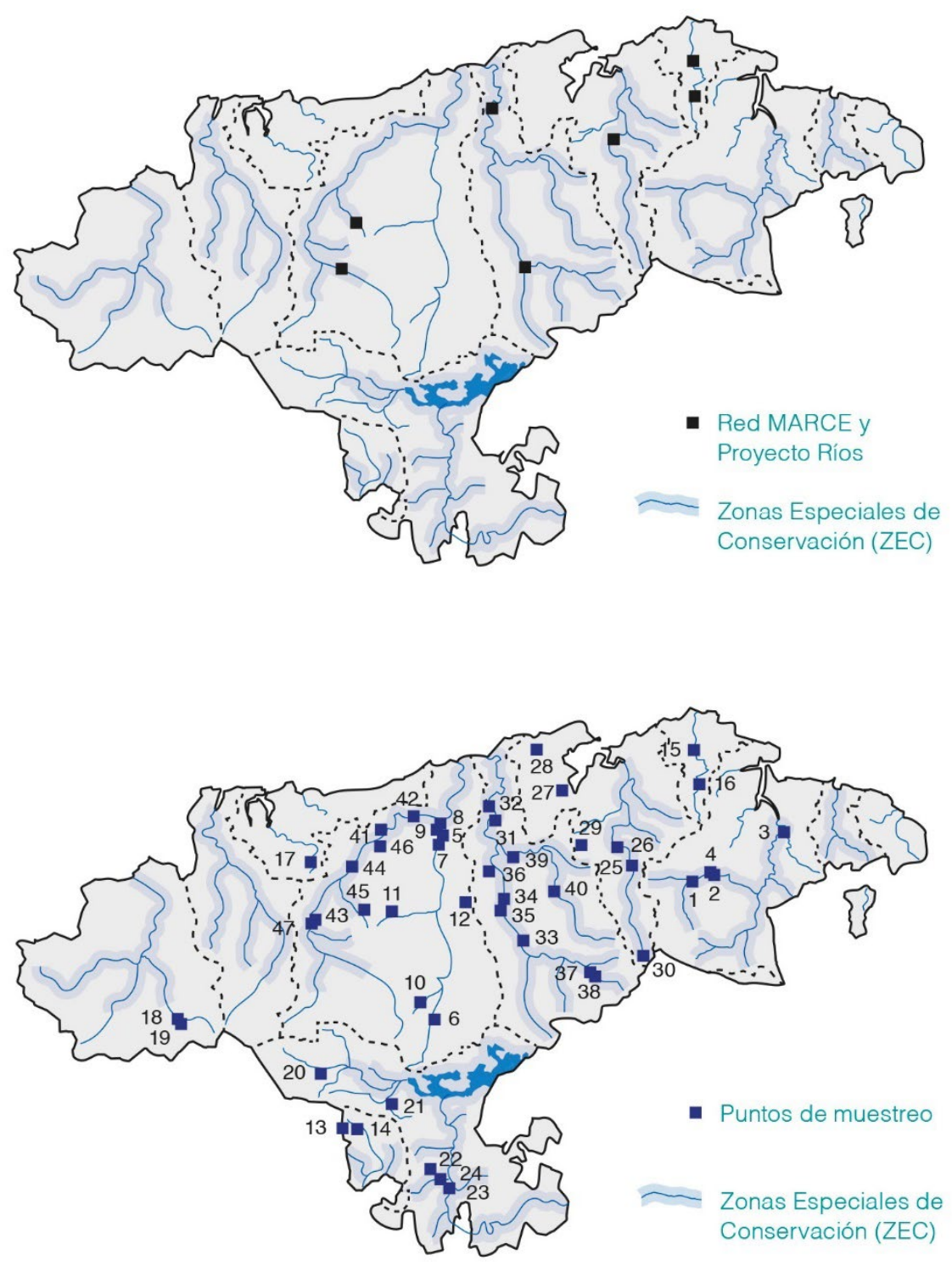

Figura 4 Puntos de muestreo de la Red MARCE localizados a una distancia menor o igual a un radio de 100 metros respecto a los puntos de muestreo de la Red Proyecto Ríos.

Figura 5 Mapa con los puntos de muestreo inspeccionados durante, al menos, 6 años en el periodo 2008 - 2017. 
Finalmente, los diagnósticos realizados en el marco del Proyecto Ríos Cantabria permitieron identificar tramos de gran valor ambiental y tramos que presentaban retos ambientales en los ríos y riberas. Alrededor de la mitad $(45.9 \%)$ de los tramos de río inspeccionados se consideraron tramos de un gran valor ambiental, presentándose como oportunidades de conservación. Por otro lado, el $54.1 \%$ (151) de los tramos inspeccionados se identificaron como retos ambientales.

En relación a los resultados del análisis a escala de punto de muestreo, 47 de los 282 tramos fueron muestreados, al menos, 6 años en el periodo 2008-2017 (Figura 5). En la Tabla 5 se muestra la evolución año a año del estado ecológico de dichos tramos inspeccionados.

\subsection{Resultados de la recopilación de acciones sociales y ambientales}

En el marco del Proyecto Ríos Cantabria (2008-2017) se realizaron las siguientes acciones:

\subsubsection{Acciones formativas}

Comprendieron salidas formativas, muestreos simultáneos, conferencias/ jornadas formativas e itinerarios interpretativos. En la Tabla 6 se detallan las actividades formativas realizadas durante la década 2008-2017.

\subsubsection{Actividades de encuentro entre personas voluntarias}

- $\quad$ Red de voluntarios: se celebraron una veintena de reuniones en diferentes cuencas cántabras con una participación de 206 personas.

- $\quad$ Romería fluvial: entre los años 2008 y 2010, se celebraron 3 romerías fluviales en el parque de Santa Lucía (Cabezón de la Sal), en Mataporquera y en el parque de Alceda (Corvera de Toranzo) respectivamente. El número total de asistentes fue de 579 personas.

\subsubsection{Proyectos de custodia fluvial y adopción de ríos}

En la Tabla 7 se detallan los resultados más destacables de los proyectos de custodia fluvial y adopción de ríos dirigidos por Red Cambera.

\subsubsection{Acciones de conservación}

En la Tabla 8 se detallan los resultados más destacables de las acciones de conservación emprendidas en el marco del Proyecto Ríos Cantabria 2008-2017.

\section{Discusión}

\subsection{0 años de datos ambientales}

El desarrollo del Proyecto Ríos ha logrado un seguimiento ambiental de la calidad biológica del agua y del estado del bosque de ribera y un diagnóstico del estado ecológico de los ríos y riberas cántabros durante
10 años. En este sentido, los diferentes resultados de los índices de calidad obtenidos a escala regional apuntan hacia una clara relación con la ubicación de los tramos. Se constatan índices de calidad más altos en los tramos altos que en los tramos medios y bajos de las cuencas cántabras, existiendo también un mayor número de tramos muestreados en las partes medias y bajas de los ríos que en las cabeceras. En este punto, cabría reflexionar acerca de la elección de los tramos que inspeccionan las personas voluntarias. En todo momento la elección del tramo es libre, y el equipo técnico de Red Cambera participa en esta fase asesorando a los nuevos grupos. Por ello, cabría considerar que en la mayoría de las ocasiones esta decisión viene directamente relacionada con la distancia del punto de muestreo respecto al núcleo de población donde residen. Este hecho conlleva la acumulación de un mayor número de puntos en las cuencas centrales de la comunidad, resultando en número inferior aquellos que se encuentran más lejanos a grandes núcleos de población, y que muy probablemente, presenten calidades superiores al contar con menores presiones antrópicas, o incluso ausencia de estas. Cabe destacar también que el perfil de los grupos de personas voluntarias que participan en el programa, aunque es muy variado (asociaciones culturales, centros de enseñanza, familias, amigos/as), dominan en gran medida los centros de enseñanza y las familias, por lo que existe una tendencia hacia la elección de tramos de fácil acceso. Este hecho puede dar lugar a un sesgo en los resultados, al no considerarse zonas de más difícil acceso,

Tabla 6 Acciones formativas: tipos, número, personas alcanzadas y, lugar y año de realización.

\begin{tabular}{|c|c|c|c|}
\hline Acciones & $\begin{array}{c}\text { № } \\
\text { acciones }\end{array}$ & $\begin{array}{c}\text { № } \\
\text { personas }\end{array}$ & Lugar y año \\
\hline $\begin{array}{l}\text { Salidas } \\
\text { formativas }\end{array}$ & 185 & 1830 & $\begin{array}{l}\text { Cuencas } \\
\text { hidrográficas } \\
\text { cántabras (2008-2017). }\end{array}$ \\
\hline $\begin{array}{l}\text { Muestreos } \\
\text { simultáneos }\end{array}$ & 4 & 64 & $\begin{array}{l}\text { Cuencas del Ebro } \\
\text { (2010), Nansa (2013), } \\
\text { Saja-Besaya (2014) y } \\
\text { Deva (2015). }\end{array}$ \\
\hline $\begin{array}{l}\text { Conferencias/ } \\
\text { Jornadas } \\
\text { formativas }\end{array}$ & 31 & 1229 & $\begin{array}{l}\text { Centro de } \\
\text { Investigación del } \\
\text { Medio Ambiente } \\
\text { (Torrelavega), } \\
\text { instalaciones de la } \\
\text { Universidad de } \\
\text { Cantabria (Santander) } \\
\text { y la Universidad } \\
\text { Nacional de } \\
\text { Educación a Distancia } \\
\text { (UNED) (2008-2017). }\end{array}$ \\
\hline $\begin{array}{l}\text { Itinerarios } \\
\text { interpretativos }\end{array}$ & 5 & 110 & $\begin{array}{l}\text { Ríos Besaya (2011), } \\
\text { Ebro (2012), Asón } \\
\text { (2013), Nansa (2013) y } \\
\text { Cubo (2013). }\end{array}$ \\
\hline
\end{tabular}


Tabla 5 Evolución temporal del estado ecológico de los puntos muestreados durante al menos 6 años en el marco del Proyecto Ríos Cantabria.

\begin{tabular}{|c|c|c|c|c|c|c|c|c|c|c|c|c|c|c|}
\hline \multirow[b]{2}{*}{ Id } & \multirow[b]{2}{*}{ Cuenca } & \multirow[b]{2}{*}{ Río } & \multirow[b]{2}{*}{ Localidad } & \multirow[b]{2}{*}{ Municipio } & \multicolumn{10}{|c|}{ Años } \\
\hline & & & & & 08 & 09 & 10 & 11 & 12 & 13 & 14 & 15 & 16 & 17 \\
\hline 1 & Asón & Asón & Arredondo & Arredondo & & & & & & & & & & \\
\hline 2 & Asón & Asón & Riva & Ruesga & & & & & & & & & & \\
\hline 3 & Asón & Asón & Marrón & Ampuero & & & & & & & & & & \\
\hline 4 & Asón & Asón & Riva & Ruesga & & & & & & & & & & \\
\hline 5 & Besaya & Besaya & Corrales de Buelna & Corrales de Buelna & & & & & & & & & & \\
\hline 6 & Besaya & Besaya & Ventorrillo & Pesquera & & & & & & & & & & \\
\hline 7 & Besaya & Besaya & Cartes & Cartes & & & & & & & & & & \\
\hline 8 & Besaya & Besaya & Parque de la Viesca & Torrelavega & & & & & & & & & & \\
\hline 9 & Besaya & La Viesca & Parque de la Viesca & Torrelavega & & & & & & & & & & \\
\hline 10 & Besaya & Bisueña & Bárcena Pie de Concha & Bárcena Pie de Concha & & & & & & & & & & \\
\hline 11 & Besaya & Cieza & Villasuso & Cieza & & & & & & & & & & \\
\hline 12 & Besaya & Tejas & Monte Tejas & San Felices de Buelna & & & & & & & & & & \\
\hline 13 & Camesa & Camesa & Reinosilla & Valdeolea & & & & & & & & & & \\
\hline 14 & Camesa & Camesa & Espinosa & Valderredible & & & & & & & & & & \\
\hline 15 & Campiazo & Campiazo & Bo Solorga & Bareyo & & & & & & & & & & \\
\hline 16 & Campiazo & Campiazo & Beranga & Hazas de Cesto & & & & & & & & & & \\
\hline 17 & Costa Oeste & Escudo & San Vicente del Monte & Valdáliga & & & & & & & & & & \\
\hline 18 & Deva & Bullón & Finca Barriales & Pesaguero & & & & & & & & & & \\
\hline 19 & Deva & Vendejo & Pesaguero & Pesaguero & & & & & & & & & & \\
\hline 20 & Ebro & Híjar & Entrambasaguas & Hdad. Campoo de Suso & & & & & & & & & & \\
\hline 21 & Ebro & Marlantes & Cervatos & Campoo de Enmedio & & & & & & & & & & \\
\hline 22 & Ebro & Polla & Valdeprado del Río & Valdeprado del Río & & & & & & & & & & \\
\hline 23 & Ebro & Polla & Bárcena de Ebro & Valdeprado del Río & & & & & & & & & & \\
\hline 24 & Ebro & Polla & Reocín de los Molinos & Valdeprado del Río & & & & & & & & & & \\
\hline 25 & Miera & Miera & Mirones & Miera & & & & & & & & & & \\
\hline 26 & Miera & Miera & Rubalcaba & Liérganes & & & & & & & & & & \\
\hline 27 & Miera & La Canaluca & El Astillero & El Astillero & & & & & & & & & & \\
\hline 28 & Miera & Otero & Sancibrián & Santa Cruz de Bezana & & & & & & & & & & \\
\hline 29 & Miera & Ozadera & Llanos & Penagos & & & & & & & & & & \\
\hline 30 & Miera & Valbuena & La Concha & San Roque de Riomiera & & & & & & & & & & \\
\hline 31 & Pas & Pas & Renedo & Piélagos & & & & & & & & & & \\
\hline 32 & Pas & Pas & Salcedo & Piélagos & & & & & & & & & & \\
\hline 33 & Pas & Pas & Alceda & Corvera de Toranzo & & & & & & & & & & \\
\hline 34 & Pas & Pas & Prases & Santiurde de Toranzo & & & & & & & & & & \\
\hline 35 & Pas & La Torcía & Borleña & Corvera de Toranzo & & & & & & & & & & \\
\hline 36 & Pas & Moro & Aes & Puente Viesgo & & & & & & & & & & \\
\hline 37 & Pas & Yera & Yera & Vega de Pas & & & & & & & & & & \\
\hline 38 & Pas & Yera & Yera & Vega de Pas & & & & & & & & & & \\
\hline 39 & Pisueña & Pisueña & Pornaluengo & Castañeda & & & & & & & & & & \\
\hline 40 & Pisueña & Pisueña & Vega & Villafufre & & & & & & & & & & \\
\hline 41 & Saja & Saja & Casar de Periedo & Cabezón de la Sal & & & & & & & & & & \\
\hline 42 & Saja & Saja & Santa Isabel de Quijas & Reocín & & & & & & & & & & \\
\hline 43 & Saja & Saja & Terán & Cabuérniga & & & & & & & & & & \\
\hline 44 & Saja & Saja & Hoz de Santa Lucía & Mazcuerras & & & & & & & & & & \\
\hline 45 & Saja & Bayones & Ucieda & Ruente & & & & & & & & & & \\
\hline 46 & Saja & Ceceja & Riaño de Ibio & Mazcuerras & & & & & & & & & & \\
\hline 47 & Saja & Pulero & Mazcuerras & Mazcuerras & & & & & & & & & & \\
\hline \multicolumn{15}{|c|}{ Código de color: } \\
\hline & Malo & & ficiente & Moderado & Buen & & & & & & & eno & & \\
\hline
\end{tabular}


Tabla 7 Entidad, duración, lugar y principales resultados de la custodia y la adopción fluvial.

\begin{tabular}{lll}
\hline Entidad & Duración & Lugar \\
Asociación & $2011-2013$ & Varios tramos de los ríos Saja y \\
Conservación y & & Besaya (Los Corrales de Buelna, \\
Biodiversidad & & San Felices de Buelna, Cabezón de \\
& la Sal y Mazcuerras).
\end{tabular}

\section{Resultados}

88 personas voluntarias involucradas.

$325 \mathrm{~kg}$. de bulbos y plantas de la especie Vara de San

José (Crocosmia x crocosmiiflora) eliminados.

Plantación de pies de: sauce cenizo (Salix

atrocinera), sauce blanco (Salix alba), mimbrera

(Salix fragilis), avellano (Corylus avellana), fresno

(Fraxinus excelsior) y arce silvestre (Acer

campestre).

$\begin{array}{ll}\begin{array}{l}\text { Propietarios de la } \\ \text { finca }\end{array} \quad 2011-2015 & \begin{array}{l}\text { Finca ganadera en Cabezón de } \\ \text { Liébana }\end{array}\end{array}$

25 personas voluntarias involucradas.

Una charca para anfibios construida y acondicionada.

Un informe de resultados de la monitorización de la

colonización de la charca.

Asociación Arcera 2012-2013 Un tramo del río Ebro (Arcera)

52 personas involucradas.

Un inventario botánico y faunístico del área de influencia.

Una jornada de limpieza del molino de La Fábrica.

Un estudio de adaptación de indicadores funcionales

y sustratos artificiales para la detección de impactos

antrópicos en el Ebro.

$\begin{array}{lll}\begin{array}{l}\text { Asociación de } \\ \text { Mujeres Jolanta }\end{array} & 2013 & \text { Un tramo del río Cabo (Polanco) } \\ \begin{array}{lll}\text { Confederación } & \text { (2014- en } & \text { Varios tramos del río Camesa } \\ \text { Hidrográfica del } & \text { vigor) } & \text { (Brañosera y Valdeolea) }\end{array}\end{array}$

24 personas involucradas.

Una plantación de especies de árboles y arbustos autóctonos.

39 personas alcanzadas.

10 trampas huella instaladas para detectar la presencia de visón americano (Neovison vison).

15 personas formadas en el uso de sustratos artificiales como método complementario al muestreo mediante el índice IBMWP.

Un inventario de las alteraciones de la continuidad hidráulica.

Un informe de resultados del seguimiento de la demolición de un azud.

que muy probablemente presenten mejores condiciones ambientales para todos los índices de calidad analizados en el Proyecto Ríos.

En relación a la validación llevada a cabo comparando los datos obtenidos por la red de personas voluntarias en el marco del Proyecto Ríos y aquellos obtenidos por el personal técnico del IH Cantabria presentan un grado de acuerdo bueno. Este hecho aporta, si cabe, aún más valor a la serie de datos obtenida por parte de las personas voluntarias en el periodo 2008- 2017. Sin embargo, el hecho que sólo 7 puntos coincidiesen para ambas redes de muestreo supone un número muy bajo si se tiene en cuenta que se han muestreado 282 tramos por parte de las personas voluntarias.

Tanto la identificación de tramos de río de gran valor ecológico como de tramos con retos ambientales, entre los tramos de río inspeccionados en el marco del Proyecto Ríos 2008-2017, supone un punto de partida para grupos de personas voluntarias que no hayan avanzado hacia la quinta y última fase del proyecto: la adopción.

\subsection{Proyecto Ríos como modelo de aprendizaje participativo}

Hasta hace poco tiempo, la ciencia ciudadana consideraba a las personas voluntarias como fuente de toma de datos (Betancur y Cañón, 2016). Sin embargo, según Olin-Scheller y Wikström (2010), se ha puesto de manifiesto que aquellos ciudadanos y ciudadanas que participan en procesos de ciencia ciudadana son capaces de tomar decisiones políticas más instruidas, logrando alzar la voz en procesos de toma de decisiones. Esto es, logran ser parte de la producción de conocimiento, así como de su consumo. De este modo, fruto del intercambio de datos entre la comunidad científica y la ciudadanía en general, se logra que las ciencias ambientales sean asequibles para todos los públicos y que la comunidad científica descubra el valor de los conocimientos desde el punto de vista de los usuarios y usuarias del medio (Figura 6). 
Tabla 8 Acciones de conservación emprendidas, lugar, año y principales resultados obtenidos.

\begin{tabular}{|c|c|c|}
\hline Actividades & Lugar y año & Resultados \\
\hline $\begin{array}{l}\text { Campo de } \\
\text { voluntariado } \\
\text { en el río Ebro }\end{array}$ & $\begin{array}{l}\text { Polientes } \\
(2010)\end{array}$ & $\begin{array}{l}20 \text { personas formadas en } \\
\text { el diagnóstico del estado } \\
\text { de los ríos a través de la } \\
\text { aplicación de índices y } \\
\text { metodologías } \\
\text { estandarizadas. }\end{array}$ \\
\hline Robinia & $\begin{array}{l}\text { Los Corrales de } \\
\text { Buelna, San } \\
\text { Felices de } \\
\text { Buelna, } \\
\text { Cabezón de la } \\
\text { Sal y } \\
\text { Mazcuerras } \\
(2011-2013)\end{array}$ & $\begin{array}{l}67 \text { responsables y } \\
\text { trabajadores de } \\
\text { entidades locales } \\
\text { formados en la } \\
\text { prevención y control de } \\
\text { especies de flora exótica } \\
\text { invasora. }\end{array}$ \\
\hline Galemys & $\begin{array}{l}\text { Cuencas de } \\
\text { Cantabria } \\
(2017)\end{array}$ & $\begin{array}{l}35 \text { personas alcanzadas y } \\
\text { formadas en el } \\
\text { seguimiento de desmán } \\
\text { ibérico (Galemys } \\
\text { pyrenaicus) y musgaño } \\
\text { patiblanco (Neomys } \\
\text { fodiens). } \\
\text { Un inventario de desmán } \\
\text { ibérico (Galemys } \\
\text { pyrenaicus). } \\
\text { Un inventario de } \\
\text { musgaño patiblanco } \\
\text { (Neomys fodiens). }\end{array}$ \\
\hline
\end{tabular}

En este sentido, cabe destacar el Proyecto Ríos frente a otros programas centrados únicamente en la transmisión de conocimiento. El Proyecto Ríos trasciende la mera educación ambiental. Su diferenciación radica en el conjunto de principios pedagógicos en los que se basa. El primero de estos principios es el aprendizaje-servicio ya que el Proyecto Ríos tiene como objetivo capacitar a la ciudadanía en diversas destrezas y valores que comprenden el conocimiento del medio, pero también la aplicación de metodologías objetivas de toma de datos. Estas últimas, útiles para la realización de estudios científicos, permiten acercar la ciencia a la población.
En segundo lugar, el Proyecto Ríos se basa en un aprendizaje vivencial y significativo. Esto es, cada persona voluntaria proviene de una experiencia propia, de un contacto con los ríos diferente, con más o menos conocimiento de lo que los paisajes fluviales presentan. En consecuencia, se cuenta con la propia experiencia previa de los participantes, de las necesidades o problemas vividos o sentidos. Es fundamental contar con las vivencias de quienes ya conocen los ríos, tanto por la información que se puede obtener, como para interrelacionar esa información que se generan con estos conocimientos previos, hecho primordial para que se produzca el aprendizaje. Es importante, por tanto, asumir otras fuentes de conocimiento además de la estrictamente científica. La historia, la observación, el uso del río, la tradición y diversas fuentes documentales, pueden aportar información muy interesante acerca de lo que son, de lo que fueron o de lo que pueden llegar a ser los ambientes fluviales. Aunque no se ha desarrollado durante los 10 años de Proyecto Ríos una evaluación temporal objetiva del aprendizaje significativo de las personas voluntarias, cabe destacar que de una manera subjetiva se ha apreciado una mejoría, en términos generales, en la calidad de la información. Dicha calidad se encuentra directamente relacionada con el número de años que las personas voluntarias participan en el programa.

En tercer lugar, el Proyecto Ríos trata de fomentar el aprendizaje entre iguales. Además del aprendizaje con personal experto (maestros/as, educadores/as, profesores/as, etc.), una fuente importante de aprendizaje proviene de las interacciones con los otros que nos son próximos (familia, amigos/as, compañeros/as, colegas, etc.). Cada persona que participa en el Proyecto Ríos tiene unos potenciales diferentes cuya puesta en común genera un enriquecimiento del grupo. Se trata de compartir, conversar, intercambiar opiniones, consultar, etc. Esto es, desarrollar una serie de habilidades sociales "mutuas" para la comprensión y resolución de la problemática ambiental.

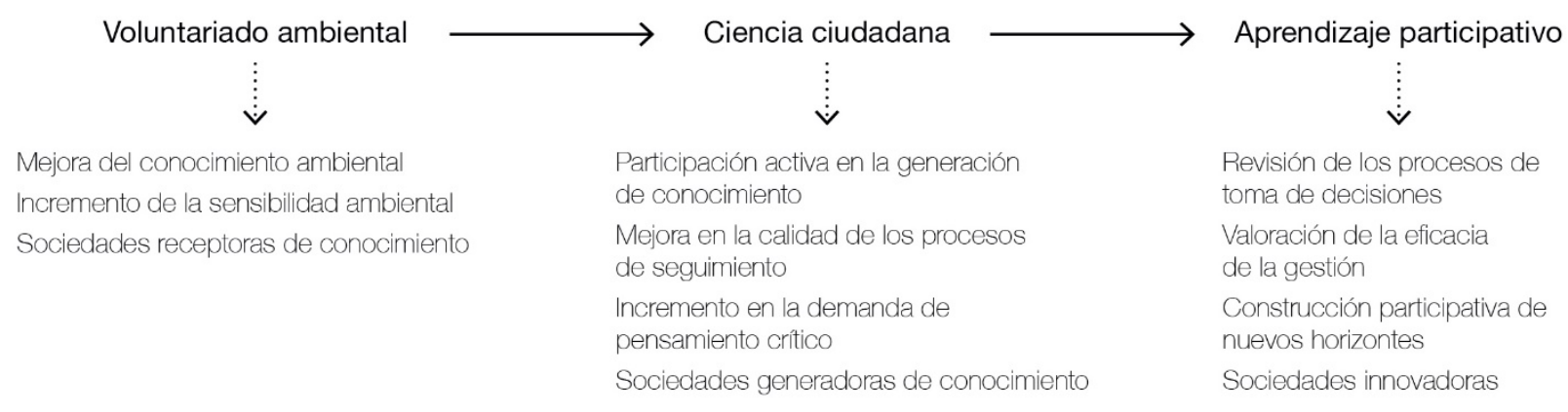

Figura 6 Relación entre el voluntariado ambiental, la ciencia ciudadana y el aprendizaje participativo. 
Finalmente, y en la línea del principio anterior, un método complementario al aprendizaje con un experto o experta, lo constituye el aprendizaje cooperativo. Este, consiste en la interconexión de los aprendizajes de los componentes del grupo de personas voluntarias para realizar la tarea de inspección, y la capacitación de los unos a los otros. Durante las diversas fases del Proyecto Ríos se articula la enseñanza en torno al aprendizaje cooperativo, es decir, todos los miembros de un mismo grupo de voluntarios y voluntarias $\mathrm{y}$, entre diversos grupos, colaboran para lograr que el fin de su tarea resulte óptima y positiva.

\subsection{Directiva Marco del Agua y Proyecto Ríos}

En el marco de la participación pública contemplado en la Directiva Marco del Agua (Directiva 2000/60/CE) (en adelante, DMA), el Proyecto Ríos proporciona a las personas voluntarias los conocimientos y destrezas necesarias para comprender el estado de los ríos y riberas de Cantabria y por lo tanto les proporciona herramientas para poder intervenir en los procesos de participación pública.

Por otro lado, la DMA nació con la vocación de garantizar la protección de los ecosistemas acuáticos y promover un uso sostenible que garantice la disponibilidad del recurso natural a largo plazo. Para ello, promueve la vigilancia sistemática para la gestión y mejora de los sistemas fluviales. Es por ello que, tanto el inventario de las presiones e impactos, como el seguimiento de los ecosistemas acuáticos, son la base para el enunciado de medidas de gestión de las masas de agua en el marco de los planes de gestión de las diferentes confederaciones hidrográficas. En este contexto, el Proyecto Ríos, aporta un seguimiento semestral de un elevado número de tramos de río localizados en las cuencas cántabras (cerca de 300), durante largos periodos de tiempo (alrededor de 50 tramos han sido diagnosticados durante más de 6 años consecutivos) (Tabla 5). Dicha información se difunde desde el año 2008 mediante la elaboración de un Informe Anual que se traslada a todas las autoridades competentes, prensa, grupos de interés y sociedad, en general. En este ámbito, se tiene constancia que algunos de los datos ambientales obtenidos en el marco del Proyecto Ríos han sido empleados como apoyo en la gestión (p.e. presencia de especies exóticas e invasoras, vertidos, etc.).

\subsection{La adopción de ríos, como instrumento de conservación ambiental}

Emprender un proyecto de adopción surge de la convicción de un grupo de personas voluntarias ilusionadas por conservar o mejorar una situación inicial. La búsqueda de un mejor estado de conservación y la ilusión de contribuir al cambio son el motor que estimula el desarrollo de ideas que, bien meditadas y estructuradas, dan lugar a buenos proyectos. En este caso, el apoyo institucional por parte de entidades como Red Cambera juega un importante papel como facilitador de recursos durante todo el proceso y como elemento aglutinador de todas las partes implicadas en la gestión. En este ámbito, uno de los mayores hitos de Red Cambera en el campo de la adopción ha sido la acontecida en varios tramos del río Camesa (Tabla 7), donde la iniciativa de la gente de la zona permitió la planificación de medidas de mejora del entorno fluvial del río Camesa, y en la que la Confederación Hidrográfica del Duero (CHD) se implicó directamente en la consecución de las medidas solicitadas por la ciudadanía para la gestión del espacio fluvial.

\section{$5 \quad$ Conclusiones}

Tras 10 años de vida, el Proyecto Ríos en Cantabria ha demostrado ser una herramienta fundamental para involucrar a la ciudadanía en el estudio y cuidado de los ríos de Cantabria. Además, ha permitido generar datos útiles como apoyo en la toma de decisiones por parte de las asociaciones conservacionistas, la comunidad científica y las administraciones públicas. A continuación, se resumen a modo de conclusiones los logros del Proyecto Ríos, las lecciones aprendidas y las oportunidades detectadas en su implementación en Cantabria durante el periodo 2008 -2017.

\section{$5.1 \quad$ Logros ambientales}

- Obtención de un diagnóstico anual del estado ecológico de los ríos y riberas cántabros durante 10 años en 282 tramos fluviales.

- Generación de datos continuos en el tiempo sobre características esenciales del ecosistema fluvial.

- Identificación de tramos de río de gran valor ecológico, cuya gestión debería abordarse para lograr la conservación de los ecosistemas fluviales.

- Identificación de tramos de río con retos ambientales, cuya gestión debería abordarse para lograr una restauración del entorno fluvial.

\subsection{Incidencia social}

- $\quad$ Participación de más de 2.400 personas.

- Participación aproximada de 500 personas voluntarias en cada campaña de muestreo.

- Implicación de más de 30 centros educativos.

- Colaboración de más de 100 entidades (asociaciones, centros educativos, administración, empresas, etc.). 


\subsection{Lecciones aprendidas}

- Conseguir involucrar a la ciudadanía en la gestión del medio fluvial resulta de vital importancia. El conocimiento del funcionamiento de los ríos, a través de la metodología del Proyecto Ríos, permite mejorar la toma de decisiones y proponer acciones de mejora en las que las personas puedan ser partícipes durante todo el proceso.

- $\quad$ El voluntariado concebido en el Proyecto Ríos es una herramienta de participación activa, que trasciende la mera consulta o suministro de información. En este sentido, las personas voluntarias construyen el Proyecto Ríos y dirigen las acciones de conservación y cuidado de ríos y riberas.

- $\quad$ El Proyecto Ríos ofrece soluciones innovadoras, inclusivas y acordadas entre todos los agentes competentes en el territorio. A través del diálogo y el consenso, se busca que las ideas puedan transformarse en proyectos que repercutan en la mejora de los ríos.

- $\quad$ El acompañamiento y comunicación continua a todos los actores implicados en cualquier iniciativa que mejore los ríos y riberas es vital para garantizar su éxito. Los procesos de participación social requieren continuidad temporal.

\subsection{Oportunidades}

- $\quad$ Posibilita la mejora de la educación ambiental efectiva, posibilitando el enunciado y desarrollo de acciones realistas y viables para la mejora y conservación del ecosistema fluvial.

- $\quad$ Aporta una metodología que permite un notable suministro de información para mejorar la gestión de las ZEC fluviales.

- $\quad$ Constituye una forma de implicar a la ciudadanía en la toma de decisiones, permitiendo la comprensión de la gestión de ríos y riberas.

\section{Agradecimientos}

Los autores quieren dar las gracias a todas las personas voluntarias del Proyecto Ríos que hacen realidad este proyecto y al Centro de Investigación del Medio Ambiente (CIMA) del Gobierno de Cantabria.

\section{$7 \quad$ Referencias}

Alba-Tercedor, J, Sánchez-Ortega, A. (1988). Un método rápido y simple para evaluar la calidad biológica de las aguas corrientes basado en el de Hellawell (1978). Limnetica, 4, 51-56.

Alonso, B. (2010). Historia de la educación ambiental. La Educación Ambiental en el Siglo XX. Asociación Española de Educación Ambiental.

Bald, J. (2005). Propuesta para la evaluación del estado físico- químico de las aguas costeras y de transición del País Vasco. Ph.D.
Thesis. Universidad de Navarra, 261 pp.

Betancur E, Cañón, J. (2016). La ciencia ciudadana como herramienta de aprendizaje significativo en educación para la conservación de la biodiversidad en Colombia. Revista científica en Ciencias Ambientales y Sostenibilidad, 3(02), 1-15.

Cloux I, Valdor P.F, Gracia A, Tejón S. (2018). 10 años de Proyecto Ríos en Cantabria. Análisis ambiental 2008 - 2017. Centro de Investigación del Medio Ambiente (CIMA). Consejería de Universidades e Investigación, Medio Ambiente y Política Social. Gobierno de Cantabria.

(Diciembre 2018; https://redcambera.org/wpcontent/uploads/2019/02/informe_PR10.pdf)

Cloux I, Valdor P, Gracia A, Tejón S. (2019). Manual de inspección de ríos. Centro de Investigación del Medio Ambiente (CIMA). Consejería de Universidades e Investigación, Medio Ambiente y Política Social. Gobierno de Cantabria.

(Abril 2019; https://redcambera.org/wpcontent/uploads/2019/04/manual_inspeccion_2019.pdf)

Cohen J. (1960). A coefficient of agreement for nominal scales. Educational and Psychological Measurement, 37- 46.

Cohen J. (1968). Weighted kappa: Nominal scale agreement with provision for scaled disagreement or partial credit. Psychological Bulletin, 70, 213-220.

Directiva 2000/60/CE del Parlamento Europeo y del Consejo, de 23 de octubre de 2000, por la que se establece un marco comunitario de actuación en el ámbito de la política de aguas (DO L 327 de 22.12.2000, pp. 1-73).

IH Cantabria (2012). Red MARCE. Diseño de un marco espacial para la gestión integrada de cuenca.

Ministerio para la Transición Ecológica (2019). Buenas prácticas en actuaciones de conservación, mantenimiento y mejora de cauces.

(2019; https://www.miteco.gob.es/es/agua/temas/gestion-delos-riesgos-de-inundacion/guia-buenas-practicas-enactuaciones-conservacion-mantenimiento-mejoracauces_tcm30-503733.pdf)

Olin-Scheller C, Wikström P. (2010). Literary prosumers: young people's reading and writing in a new media landscape. Education Inquiry, 1(1), 41-56.

Prat N, Munné A, Rieradevall M, Solà C, Bonada N. (2000). ECOSTRIMED. Protocol per determinar l'estat ecològic dels rius mediterranis. Diputació de Barcelona. Área de Medi Ambient. Estudis de la Qualitat Ecològica dels Rius 80, 94-94.

Serrano F, Holocher-Ertl T, Kieslinger B, Sanz F, Silva CG. (2014). White Paper on Citizen Science in Europe. Socientize Consortium. European Commission.

(Abril 2019;

http://www.zsi.at/object/project/2340/attach/White_PaperFinal-Print.pdf).

Suárez ML, Vidal-Abarca MR, Sanchez-Montoya M, Alba-Tercedor J Alvarez M, Aviles J, Bonada N, Casas J, Jáimez-Cuellar P, Munné A, Pardo I, Prat N, Rieradevall M, Salinas MJ, Toro M, Vivas S. (2002). Las riberas de los ríos mediterráneos y su calidad: El uso del índice QBR. Limnetica, 21, 135-148.

Unión Internacional para la Conservación de la Naturaleza (UICN). (1970). Carta de Nevada. Nevada, E.U: UICN. 